\title{
Countering COVID-19 vaccine hesitancy
}

\author{
Cody Perry BS, Adin Mizer BS, Adam Wynn BS, Cassie Kruczek PhD
}

\begin{abstract}
Introduction: Many devastating diseases have been largely controlled or eradicated, especially in industrialized nations, due to the availability of safe, effective, and affordable vaccines. The COVID-19 pandemic has resulted in a horrific toll on human life and is devastating the global economy. To prevent the continued spread of COVID-19, efforts have begun to develop a COVID-19 vaccine. However, recent public polls have demonstrated concerns with the COVID-19 vaccine. To help understand these concerns, this review addresses the necessity of vaccines, vaccine safety and development, and vaccine hesitancy.

Methods: This review contains a compilation of data from several sources, including peerreviewed journals, preprints, studies published in PubMed and the Cochrane Library, CDC and WHO guidelines, and broader web searches to retrieve up-to-date information. Studies selected were related to vaccine safety, production, and efficacy or COVID-19.

Results/Conclusion: To improve public acceptance of a COVID-19 vaccine, public health officials and healthcare professionals need to work with community leaders to create specialized strategies to overcome vaccine hesitancy. Such strategies could use both traditional and social media platforms in addition to physician-patient interactions. To maximize the number of individuals who receive the COVID-19 vaccine, these strategies should focus on dispelling potential myths and emphasize the benefits and safety of vaccination.
\end{abstract}

Keywords: COVID-19, SARS-CoV-2, vaccine, vaccine hesitancy, vaccine safety/efficacy

\section{INTRODUCTION}

Before the advent and regular administration of vaccines, infectious diseases, such as measles, diphtheria, polio, smallpox, and pertussis, were leading causes of childhood mortality. These diseases regularly devastated families and communities, but fortunately many of these illnesses have been contained through the development and widespread distribution of safe, effective, and affordable vaccines. ${ }^{1}$ Despite the dramatic reduction in morbidity and mortality afforded by vaccination, current vaccines provide little to no protection against variant or novel strains of infectious viruses. In

Corresponding author: Cassie Kruczek

Contact Information: Cassie.Kruczek@ttuhsc.edu

DOI: 10.12746/swrccc.v8i36.749
December 2019, the novel coronavirus (CoV) SARSCoV-2 emerged in Wuhan, China and soon spread worldwide. This virus causes the condition referred to as "coronavirus disease 2019 (COVID-19)".2,3

Coronaviruses are enveloped, positive-sense single-stranded RNA viruses ((+) ssRNA virus) belonging to the family Coronaviridae. ${ }^{4}$ Many coronaviruses are known to infect humans. For example, 15-30\% of common colds are caused by human coronaviruses (HCoVs). ${ }^{5}$ However, many coronaviruses are also zoonotic, which means that they primarily infect various animals. These zoonotic coronaviruses may be transmitted from animal reservoirs, such as bats, through an intermediate host (e.g., civet cats, camels, pangolins) to humans causing outbreaks, as seen during the severe acute respiratory syndrome (SARS) outbreak in 2002, the Middle East respiratory syndrome (MERS) outbreak in 2012, and now during the current 
COVID-19 pandemic..$^{6-10}$ When these zoonotic viruses infect humans, particularly those in older age groups with comorbidities or in patients with relevant risk factors (e.g., hypertension, allergic diseases, asthma, COPD), they can cause severe respiratory illnesses, such as acute respiratory distress syndrome (ARDS) and pneumonia, leading to death. ${ }^{11-14}$

Since the onset of the COVID-19 pandemic, numerous tactics, such as increased emphasis on hand hygiene, social distancing, the donning of face coverings and gloves by the general public, and government stay-at-home orders have been used to reduce the spread of COVID-19. ${ }^{15-17}$ In spite of these efforts, SARS-CoV-2 has continued to spread, and as of July 14,2020 , it has resulted in over 13 million confirmed cases of COVID-19 and more than 570,000 deaths worldwide. ${ }^{18}$ In addition to this toll on human life, this disease has disrupted workplaces, schools, and economies and strained healthcare systems with severe consequences. The total number of cases and fatalities from this deadly plague continues to climb daily, and some models suggest a potential resurgence of SARS-CoV-2 during the winter. These models also warn that without appropriate measures, such as intermittent social distancing, widespread surveillance, new therapeutics, and vaccines, critical care capacity may again be exceeded, and healthcare systems overwhelmed. ${ }^{19,20}$ As there are no specific therapeutics or vaccines in place to control COVID-19, attention has shifted toward preventing this disease and other potential future resurgences of the virus through the research and development of a COVID-19 vaccine.${ }^{21}$ Currently, through the Accelerating COVID19 Therapeutic Interventions and Vaccines (ACTIV) public-private partnership, numerous government agencies, including the National Institutes of Health $(\mathrm{NIH})$, the Food and Drug Administration (FDA), and the Centers for Disease Control and Prevention (CDC), have partnered with representatives from academia, philanthropic organizations, and more than 15 biopharmaceutical companies to develop a safe and effective COVID-19 vaccine. ${ }^{21,22}$

A poll recently conducted in the United States reported that if a vaccine against COVID-19 were to become available to the public, $49 \%$ of those surveyed plan to receive it, $31 \%$ were unsure, and $20 \%$ said they would not. ${ }^{23}$ These results, combined with the fact that an increasing number of people are requesting alternative vaccination schedules or declining vaccination altogether, provide evidence that COVID19 vaccine hesitancy has already become an issue that needs to be addressed. ${ }^{24}$

Since the general public may have questions and concerns about the COVID-19 vaccine, this literature review will provide information on vaccine immunology and typing and will then examine the need for vaccines, vaccine safety and development, and vaccine hesitancy. ${ }^{22,25}$ To return to a semblance of previous normality, a COVID-19 vaccine is an absolute necessity, and adequate preparation from healthcare professionals, local communities, and government organizations will be required to ensure the successful delivery of this crucial vaccine. ${ }^{21}$

\section{Methods}

This review contains a compilation of data from several sources, including peer-reviewed journals, preprints, studies listed in PubMed and the Cochrane Library, CDC and WHO guidelines, and broader web searches to retrieve up-to-date information. Studies selected were related to vaccine safety, production, and efficacy or COVID-19.

\section{VACCINE IMMUNOLOGY AND TYPING}

To understand how vaccines reduce the risk of disease, it helps to first understand host defenses against infections. Pathogens, such as bacteria and viruses, cause infection when they invade the body. If unopposed, these pathogens can replicate and cause tissue damage and disease. The immune system counters these invasions with white blood cells, such as $B$ and $T$ lymphocytes. ${ }^{26}$

Following the first exposure to a pathogen, it can take the immune system several days to weeks to activate the cells of adaptive immunity required to eliminate the pathogen. Before activation of antigen specific immune cells, the pathogen can replicate and cause damage to the host. To reduce further damage from subsequent reinfection, the body produces 


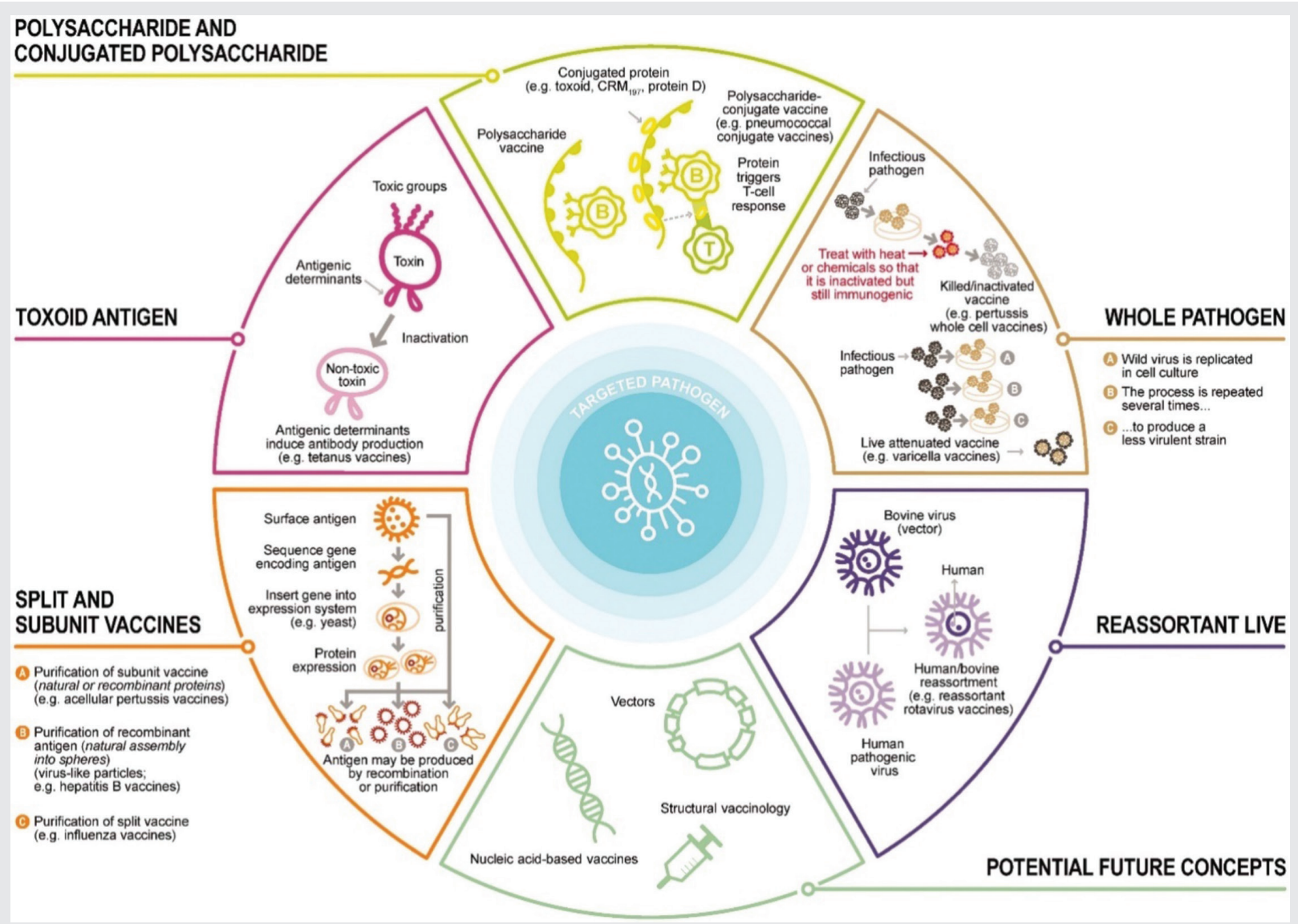

Figure 1. Different Types of Vaccines. Vaccines can be produced using different antigens. Vaccines may contain live attenuated pathogens (usually viruses), inactivated whole pathogens, toxoids (an inactivated form of the toxin produced by bacteria that causes the disease), or parts of the pathogens (e.g., natural or recombinant proteins, polysaccharides, conjugated polysaccharide or virus-like particles). ${ }^{27} \mathrm{https}$ ://www.tandfonline.com/doi/full/10.1080/07853890.2017.1407035

long-living, antigen specific "memory" T cells and antibody secreting B cells. These cells quickly recognize and eliminate the pathogen during reinfection. ${ }^{26}$

Essentially, vaccines contain attenuated versions of these disease-causing pathogens or their components, and vaccination allows patients to build immunological memory without acquiring a natural infection. Now the immune system is primed to quickly recognize and eliminate pathogens should they re-infect the host. ${ }^{26}$

Principles of vaccine immunology provide a framework for understanding how various vaccine types elicit a protective response. Although healthcare providers are aware of vaccine preventable diseases, many have not received adequate education about the different vaccine types. A summary of commonly used vaccine formulations is provided in Figure $1 .{ }^{27}$ In addition, many younger physicians have not encountered patients with some vaccine-preventable diseases that were prevalent in years past. This may account, in part, for younger physicians being receptive to modified vaccine schedules, whereas their older counterparts, who have practiced for several decades and have witnessed vaccine-preventable diseases firsthand, tend to advocate strongly for vaccinations according to recommended schedules. ${ }^{28}$ Thus, the combination of inadequate education on vaccines and inexperience with diseases may contribute to vaccine hesitancy in both healthcare providers and their patients. ${ }^{27,28}$ 
Despite the proven track record for commonly used vaccine types (e.g., live attenuated, inactivated, toxoid), scientists believe that the wide geographic diversity of the COVID-19 pandemic requires more than one effective vaccine approach. ${ }^{29,30}$ Currently, more advanced techniques, such as vectored vaccine and nucleic acid-based approaches, are being studied by biopharmaceutical companies to meet this need. ${ }^{2,21,27}$

A major target of COVID-19 vaccines is the spike (S1) glycoprotein, which is a critical virulence factor that enables coronaviruses to bind the human angiotensin-converting enzyme 2 (ACE2) receptor and infect host cells. ${ }^{31}$ To promote recognition of proteins, such as the S1 glycoprotein, viral vector delivery vaccines can be used. Vector-based vaccines use viruses that have been modified to express antigenic protein genes of the target pathogen and can be derived from retroviruses, herpes simplex viruses (HSV), adenoviruses, or poxviruses. The use of vector-based vaccines can be limited by a patient's previous exposure to the viruses used as vectors, leading to high prevalence of pre-existing antibodies against the vectors. These pre-existing antibodies may cause early vaccine clearance and result in reduced immunogenicity. ${ }^{27}$ An example of this type of vaccine is the recombinant adenovirus type-5 (Ad5) vectored COVID-19 vaccine, which expresses the S1 glycoprotein. In one study, 108 participants received this vaccine, and although over $75 \%$ of participants experienced mild or moderate adverse reactions, such as pain at the injection site (54\%), fever (46\%), fatigue $(44 \%)$, headache $(39 \%)$, and muscle pain $(17 \%)$, no serious adverse events were reported. In addition, at day 14 post-vaccination, neutralizing antibodies increased significantly, and rapid, antigen-specific $T$ cell responses were noted, suggesting that the vaccine has the potential to be highly effective. ${ }^{32}$

Nucleic acid-based vaccines are also being studied to prevent COVID-19 infections. ${ }^{2}$ These vaccines work by inserting DNA or RNA that encode antigenic proteins into host cells. This leads to presentation of the antigen to $T$ and $B$ lymphocytes, triggering an immune response. ${ }^{33,34}$ Several studies have attested to the safety of both DNA- and RNA-based vaccines, yet there is a theoretical risk that the integration of introduced DNA could result in insertional mutagenesis or induced autoimmunity. ${ }^{35,36}$ Despite the reported safety record for nucleic acid vaccines, their shelf-life and stability is uncertain; research generally indicates that DNA vaccines are more stable than mRNA vaccines. $^{36-38}$ Furthermore, DNA vaccines specifically have been shown to generate protective immunity in common laboratory animals but, with a few exceptions, have not been equally effective in humans. ${ }^{36}$ In contrast, some pilot clinical trials showed that dendritic cells transfected with mRNA vaccines encoding HIV-1 antigens, cellular molecules, or pp65 of human cytomegalovirus elicited antigen-specific CD4 and CD8 $T$ cell immune responses, but no reduction in viral load was observed. ${ }^{39-42}$ Another recently conducted clinical trial of a protamine-complexed mRNA vaccine against rabies virus showed that the vaccine induced a robust humoral immune response in volunteers, as long as it was administered with a needle-free device. ${ }^{43}$ These successes with mRNA vaccines have led to the development of an mRNA vaccine that encodes the S1 glycoprotein of SARS-CoV-2, which has also entered the clinical trial phases of development. ${ }^{2,27}$

Although vector and nucleic acid-based vaccine types have shown promise in animal studies and small clinical trials, it is essential that the producers of the COVID-19 vaccine provide timely and accurate information regarding their clinical trial results, so that the general public and medical providers can have confidence in the efficacy of these vaccines. ${ }^{21,44}$

\section{THE NECESSITY OF VACCINES}

Despite significant reductions in disease morbidity and mortality credited to vaccines, some members of the public continue to remain skeptical as to whether vaccines, including a potential COVID-19 vaccine, are actually needed. Although the arguments surrounding this topic are multifaceted, perhaps the simplest approach is to determine if vaccines work and if they are superior to the existing alternatives (e.g., immunity derived from natural infection). ${ }^{1}$

Vaccine effectiveness refers to "the protection conferred by immunization in a defined population. It measures both direct (vaccine-induced) and indirect 
(population-related) protection," e.g., herd immunity. Vaccine effectiveness is directly related to the efficacy of the vaccine, but other factors, such as vaccine coverage, may also influence effectiveness. ${ }^{45}$

The direct effect of vaccination is "the reduction in the probability of developing the disease, which is determined by comparing vaccinated and unvaccinated persons belonging to the same population." ${ }^{45} \mathrm{An}$ example of the direct effect can be observed through the administration of the Hepatitis $B$ vaccine to babies born to mothers who were positive for the Hepatitis $B$ virus (HBV). Babies born to a mother with HBV have a $70-90 \%$ risk of developing chronic HBV infection; approximately $25 \%$ of these children may develop severe liver disease later in life. ${ }^{46-48}$ However, a longitudinal study was performed in which children born to mothers at high risk for HBV transmission were vaccinated with the HBV vaccine at birth and at 1,2 , and 12 months post-birth. Blood samples collected yearly for 20 years demonstrated that none of the study subjects acquired chronic HBV infections or clinical Hepatitis B disease, indicating a significant reduction in disease risk. ${ }^{49}$ Similar reductions in incidence of disease have been observed in studies involving the Hepatitis $A$ and Haemophilus influenzae (Hib) vaccines. This effective reduction in risk and incidence of disease must be determined with COVID-19 vaccination. . $^{21,45,50}$

The indirect effect measures risk reduction for an individual who did not receive the vaccine.$^{51}$ When most of a population is immune to an infectious disease, there is reduced transmission of the infection, which in turn protects individuals who are still susceptible. ${ }^{45}$ This concept is called "herd immunity." The basic reproduction number (RO) is defined as the average number of other individuals that each infected individual will infect. In general, the higher the R0 value, the greater proportion of the population that will need to be immune, either through natural infection or immunization, to reach the protective herd immunity threshold. ${ }^{45}$ For example, the R0 for whooping cough, a disease caused by the bacterium Bordetella pertussis, is 5.5 , meaning that one individual infected with whooping cough would likely infect 5-6 other people. ${ }^{52}$ However, if $92-94 \%$ of the population is vaccinated against whooping cough, then the herd immunity threshold is reached, and the infected individual is significantly less likely to infect anyone
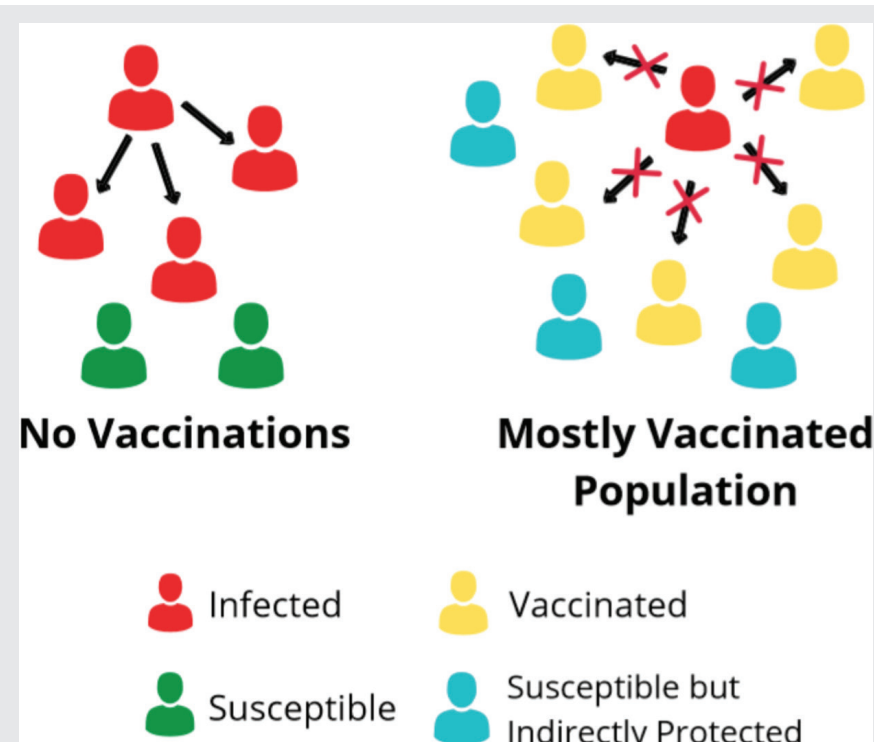

Vaccinated

Susceptible but Indirectly Protected

Figure 2. Herd Immunity. Herd immunity in a mostly vaccinated population slows the spread of disease and reduces the number of susceptible individuals and can confer protection to those who remain susceptible..$^{45,53}$ Created by authors.

else. This ultimately means that the spread of the disease is, in theory, blocked (see Figure 2).

Indirect effects of vaccine administration have been demonstrated through the rotavirus vaccine. In Austria, a universal mass rotavirus vaccine campaign began in 2007 and reached $87 \%$ coverage for children between 3 and 20 weeks of age. Following this universal mass vaccination, there was a $74 \%$ reduction in rotavirus gastroenteritis hospitalizations in the vaccinated age group, and rotavirus related hospitalizations decreased by $22 \%$ in older children (32-60 months of age) and by $47 \%$ in younger children (below 3 months of age). ${ }^{54}$ Neither of these latter two age groups were vaccinated against rotavirus, suggesting the attainment of herd immunity. ${ }^{45,54}$

The overall effect provides a broader perspective than either the direct or indirect effect alone, as it measures the "effect of immunization programs on the entire population, including both vaccinated and unvaccinated individuals." 55 This overall effect has been observed in the United States (Table 1), where 
Table 1. Impact of Vaccines in 20th and 21st Centuries

\begin{tabular}{|l|c|c|c|}
\hline Disease & $\begin{array}{c}\text { 20th Century } \\
\text { Annual Morbidity }\end{array}$ & 2017 Reported Cases & \% Decrease \\
\hline Smallpox & 29,005 & 0 & $100 \%$ \\
\hline Diphtheria & 21,053 & 0 & $100 \%$ \\
\hline Pertussis & 200,752 & 18,975 & $91 \%$ \\
\hline Tetanus & 580 & 33 & $94 \%$ \\
\hline Polio (paralytic) & 16,316 & 0 & $100 \%$ \\
\hline Measles & 530,217 & 120 & $>99 \%$ \\
\hline Mumps & 162,344 & 6,109 & $96 \%$ \\
\hline Rubella & 47,745 & 7 & $>99 \%$ \\
\hline
\end{tabular}

Table 1 from Comparison of 20th Century Annual Morbidity \& Current Morbidity: Vaccine-Preventable Diseases. ${ }^{57,58} \mathrm{https}: / /$ www.cdc.gov/vaccines/pubs/pinkbook/downloads/appendices/e/impact.pdf

widespread vaccination efforts have significantly reduced the number of cases of vaccine-preventable diseases. Thus, the direct, indirect, and overall effects of vaccination provide substantial evidence that vaccines are a highly effective method for protecting both healthy and vulnerable populations from devastating diseases. ${ }^{45,56-58}$

Vaccine hesitancy is a major barrier to vaccine uptake and could prevent the achievement of reaching the COVID-19 herd immunity threshold. Some researchers have suggested this threshold to be anywhere between $55 \%$ to $82 \%$ of the population. A vaccine refusal rate of greater than $10 \%$ from healthy individuals could obstruct attainment of this threshold, as others may be ineligible for COVID-19 vaccination due to age, immunocompromised status, and other preexisting medical conditions. ${ }^{21,22}$ Failure to achieve this threshold, compounded by the fact that pre-symptomatic carriers can affect the transmission of SARS-CoV-2, would greatly increase the susceptibility of these vulnerable populations, endangering their health. ${ }^{45,50,59,60-62}$

In addition to the benefits of vaccination in terms of protecting individuals from disease, vaccines may also provide great economic and societal benefits. Take for example the rotavirus vaccine, which prevents many cases of infant diarrhea. Although the gastroenteritis associated with a rotavirus infection may not require a visit to the doctor, a parent may have to miss work to stay home and care for the sick child. Considering that infant diarrhea caused by rotavirus can occur in up to $40 \%$ of children under 5 years of age during epidemic winter periods, this vaccine can indirectly help working parents to remain engaged in the workforce and provide for their families. ${ }^{45}$

Similar to the benefits illustrated by the rotavirus vaccine, the introduction of a COVID-19 vaccine may positively impact not only patients, but families, employers, and the economy. Since the outbreak of COVID-19, almost every sector of the economy has suffered, resulting in the United States's unemployment rate increasing from just $3.5 \%$ in February 2020 to a staggering $11.1 \%$ in June 2020 . The damage done by COVID-19 extends beyond the job market. Many schools have been closed, further widening socio-economic disparities in education and placing greater financial burdens on families. Healthcare systems, especially in large cities, have reached critical shortages in personal protective equipment (PPE), ventilators, and staff, putting both patients and healthcare providers at risk. In addition, concerns have been raised that domestic abuse and mental health disorders have increased due to lockdown and social distancing measures. These sobering reports demonstrate the negative effects on the global economy and society when left defenseless against COVID-19. Nevertheless, the successful production of a COVID19 vaccine(s) could prevent much of the economic, 
educational, and social hardships seen in recent months from reoccurring. ${ }^{63,64}$

Vaccine necessity is dependent on whether vaccines work in reducing disease burden, and if they are superior to the alternative of natural immunization. Immunity can occur when a susceptible individual is exposed to a pathogenic agent and has a protective immune response. This exposure can occur in one of two ways, either naturally (from having the disease itself), as is the case when a person is exposed to influenza, or through medical interventions, which occurs when a person receives a vaccine. Some vaccinehesitant individuals may object to vaccines on the grounds that natural immunization is better than immunization through vaccines. However, it is essential to recognize that although immunity can be obtained through either natural immunization or through vaccination, only vaccination allows the recipient to avoid exposure to diseases in a form that could cause disability or death. ${ }^{1,26}$

COVID-19 symptoms can be unpredictable, with more mild forms causing fever/chills, cough, and fatigue and more severe forms leading to acute respiratory distress syndrome (ARDS), pneumonia, and death. ${ }^{2}$ Similar to COVID-19, many vaccinepreventable diseases can either cause mild symptoms or severe complications depending on the individual infected. Therefore, a major benefit of vaccination is not just the buildup of immunity but also the reduction in severe manifestations of disease. ${ }^{26,65}$

Although it is true that natural immunity usually leads to better long-term immunity than vaccines, this is not always the case, as some vaccines (e.g., the human papillomavirus [HPV] vaccine) have been reported to induce a better immune response than natural infections. ${ }^{66}$ In addition, vaccines, especially when a booster regiment is followed, can generate sufficient long-term immunity. ${ }^{67-70}$

Contrary to what one might expect, natural infection from COVID-19 may not generate a sufficient long-term immune response, as a recent study found that some patients recovered from COVID-19 have insufficient levels of protective antibodies. However, researchers were also able to sequence antibodies that are specific to the SARS-CoV-2 spike protein that possess high neutralizing capabilities. Thus, a vaccine designed to elicit these antibodies could be broadly effective and provide greater immunity than natural infection. ${ }^{71}$

\section{VACCINE SAFETY AND DEVELOPMENT}

Since the development of the first smallpox vaccines, vaccine hesitancy has existed due to concerns over vaccine safety. These same concerns still exist today. For example, allegations have been made that vaccines are linked to autism and other learning disabilities. ${ }^{72}$ Although these claims have been proven false, concerns over vaccine safety still remain, especially from parents. ${ }^{73,74}$ This section will address these concerns by highlighting the methods vaccine developers implement to detect adverse effects before the vaccine is available to the general public. It will also outline the precautionary steps followed in the development of all vaccines that maximize both efficacy and patient safety. ${ }^{1,62,75}$

The first step in the potential production of a vaccine is determining whether or not the targeted disease-causing pathogen warrants a vaccine and if a suitable immunogenic target exists. Feasibility can be determined by various factors, such as number of strains of the target pathogen. This is part of the reason a vaccine against rhinoviruses, which have over 200 known strains, has not yet been developed. In addition, a pathogen, such as the rhinovirus, causes annoying yet usually non-life threatening infections. Thus, a good vaccine candidate would be one that causes significant disease with a limited number of strains or conserved immunogenic regions. ${ }^{24}$

After it has been determined that a vaccine is warranted and feasible, vaccine development undergoes several stages of nonclinical and clinical testing followed by post-marketing surveillance. These phases are summarized in Table 2.

As an additional note to the information provided in Table 2, vaccines are held to a higher standard of safety than many other medical products. ${ }^{80}$ However, as with all medical products, no vaccine is perfectly safe or entirely effective. Vaccines can cause minor adverse events (AE), such as fever or local reactions at the injection site, or, on rare occasion, they can 
Table 2. Clinical Trial Phases of Vaccine Development

\begin{tabular}{|c|c|c|}
\hline Phase & Participants & Purposes \\
\hline Nonclinical Phase & $\begin{array}{l}\text { Computer modeling } \\
\text { Cell line infectivity assays } \\
\text { Animals receive test vaccine }{ }^{76} \\
\end{array}$ & $\begin{array}{l}\text { Gauge vaccine safety and efficacy before } \\
\text { administration to human subjects. }\end{array}$ \\
\hline \multicolumn{3}{|c|}{ Investigational New Drug (IND) Application sent to FDA } \\
\hline $\begin{array}{l}\text { Phase I of Clinical } \\
\text { Trials }\end{array}$ & $\begin{array}{l}20-100 \text { healthy human volunteers receive } \\
\text { test vaccine. }\end{array}$ & $\begin{array}{l}\text { Assess whether the vaccine elicits an immune response } \\
\text { in humans. } \\
\text { Determine optimal vaccine dosage and route of } \\
\text { administration. } \\
\text { Gauge vaccine safety in humans. } .^{24,77}\end{array}$ \\
\hline $\begin{array}{l}\text { Phase II of } \\
\text { Clinical Trials }\end{array}$ & $\begin{array}{l}\text { Several hundred human volunteers, many } \\
\text { of whom will have characteristics similar } \\
\text { to the target population for that particular } \\
\text { vaccine, receive either the test vaccine or } \\
\text { a placebo vaccine. }{ }^{39}\end{array}$ & $\begin{array}{l}\text { Minimize confounding variables by administering } \\
\text { either the test vaccine or a placebo-control vaccine to } \\
\text { test subjects. } \\
\text { Identify side effects in population of interest. } \\
\text { Human subjects are often exposed to the pathogen } \\
\text { under controlled conditions to test vaccine efficacy. }\end{array}$ \\
\hline $\begin{array}{l}\text { Phase III of } \\
\text { Clinical Trials }\end{array}$ & $\begin{array}{l}\text { Hundreds to thousands of at-risk human } \\
\text { volunteers receive either the test vaccine } \\
\text { or a placebo vaccine. }\end{array}$ & $\begin{array}{l}\text { Detect uncommon side effects. Test vaccine efficacy } \\
\text { under natural conditions by monitoring volunteers } \\
\text { in their natural environments after receiving the test } \\
\text { vaccine. }^{77}\end{array}$ \\
\hline \multicolumn{3}{|c|}{ Vaccine licensed for administration to the public by the FDA. } \\
\hline $\begin{array}{l}\text { Phase IV } \\
\text { (Post-marketing } \\
\text { Surveillance) }\end{array}$ & $\begin{array}{l}\text { The CDC and FDA investigate any } \\
\text { suspected correlations between vaccines } \\
\text { and unknown side effects that emerge } \\
\text { after licensure. These suspected } \\
\text { correlations can be reported by the } \\
\text { general public via the Vaccine Adverse } \\
\text { Events Reporting System (VAERS) or by } \\
\text { health organizations through the Vaccine } \\
\text { Safety Datalink (VSD). }\end{array}$ & $\begin{array}{l}\text { Allows for detection of extremely rare side effects. } \\
\text { Ensures continued safety even after licensure. }\end{array}$ \\
\hline
\end{tabular}

cause serious AEs, such as anaphylaxis. ${ }^{81}$ Of the 317 million doses of vaccine distributed in the U.S. each year, the Vaccine Adverse Events Reporting System (VAERS) receives approximately 40,588 reports of AEs following immunization. Each of these reports is accepted and entered without case-by-case determination of whether the AEs could have been caused by the vaccine in question (e.g., AEs that occur coincidentally after vaccine administration). Of the U.S. primary reports received between 2012 and 2016, 94.6\% reported non-serious $A E, 5 \%$ reported a serious nonfatal $A E$, and $0.4 \%$ reported death as the outcome. ${ }^{82}$
With the current COVID-19 pandemic and the drastic effects it has had on the lives of people around the world, the push for development and distribution of a vaccine is perhaps greater than ever. ${ }^{83}$ During this increased push, some members of the public have valid concerns as to how this process will be sped up and whether this unprecedented acceleration will compromise the safety of the COVID-19 vaccine. Currently, the United States plans to shorten this process by accelerating vaccine manufacturing and distribution. Creating a vaccine to distribute worldwide has many logistical challenges. ${ }^{84,85}$ Proper vaccine 
production machinery, protocols, and materials must be distributed to multiple manufacturing sites. After this step, much more time is required to produce the millions of doses needed to distribute to the public. Because these processes are extremely expensive, the United States generally does not begin these manufacturing steps until a viable vaccine candidate has been identified and proven safe. ${ }^{29}$ However, to accelerate COVID-19 vaccine production and distribution, the United States plans to begin these preliminary manufacturing steps while vaccines are still undergoing clinical testing. This will allow distribution to begin immediately following clinical trials, but will be extremely costly should the vaccine end up failing in clinical trials. It may be worth noting, however, that while speeding up development and distribution in this manner is financially risky, it should not compromise the safety of the eventual vaccine. ${ }^{86}$

\section{ADDRESSING OTHER FACTORS CONTRIBUTING TO VACCINE HESITANCY}

\section{Misinformation}

With the increased availability of medical information, many people are taking a more active role in their treatment decisions. This increased patient involvement can lead to improved health outcomes, as patients are more likely to follow treatment regimens that they are a partner in creating. However, potential setbacks can occur when patients acquire nonfactual information and choose the misinformation over the advice of their provider. This does not mean individuals should believe everything their doctor says without question, but rather that people should be hesitant to believe everything they read, especially concerning vaccines, on the Internet. This is especially applicable to COVID-19 information, which often has little validation. This has caused many to become wary of treatments or vaccines, largely due to a falsified association between the MMR vaccine and autism. ${ }^{73,74,87,88}$ Misinformation regarding vaccines continues to spread throughout developed nations via the Internet, particularly through blogs and social media platforms. ${ }^{28}$

With the current COVID-19 pandemic, numerous organizations are working round-the-clock to develop a vaccine, and some state governors are threatening the continuation of lockdowns until a vaccine is made available. This urgency to produce the COVID-19 vaccine has raised concerns from the public that this vaccine will not be safe and may account for some of the hesitancy surrounding the COVID-19 vaccine..$^{89,90}$ This unease is in part responsible for COVID-19 vaccine conspiracy theories on social media platforms. ${ }^{91}$ Thus, swift and prudent intervention is required to correct misinformation and increase COVID-19 vaccine acceptance. ${ }^{50}$ The diverse American population requires various platforms to effectively communicate accurate information regarding the COVID-19 vaccine. As social media can serve as a platform for both factual and false information, some experts have called for the use of health campaigns that utilize traditional and social media to counteract anti-COVID-19 vaccine messaging in real time. These public health campaigns should enlist the expertise of physicians and other frontline healthcare workers to monitor, counter, and prevent the spread of ill-conceived notions about a future COVID-19 vaccine before dangerous myths take root in the public psyche. ${ }^{22}$ However, healthcare professionals should not restrict their efforts to merely disproving vaccination myths, but emphasize the benefits of vaccination and the severity of the diseases vaccines prevent. ${ }^{92,93}$

\section{Mistrust}

In general, there is a growing distrust of the government and the healthcare system, in part due to subpopulations that have experienced historical and contemporary mistreatment and disparities in care. ${ }^{94}$ Recent reports have demonstrated that long-standing health and social inequalities have put some minority groups, such as American Indian, African American, and Hispanic or Latino populations, at increased risk of COVID-19 infection or severe illness. ${ }^{95}$ One option to rebuild public trust and promote health equity is for public health officials and medical professionals to work with community leaders outside of traditional medicine to develop and spread educational content regarding vaccines that is both readily accessible and culturally relevant. ${ }^{22}$ Public health officials should work with respected community leaders, school nurses, disease survivors, and parents, as 
studies have demonstrated higher levels of trust in these groups among minority populations. ${ }^{96}$ Working together, healthcare professionals and community leaders can educate and encourage the public to take an active role in using a potential COVID-19 vaccine to safeguard their individual health and the health of the community. ${ }^{22,97}$

\section{Concerns Regarding Mandatory VACCINATION Programs}

For decades, all 50 states have required that parents vaccinate their children as a prerequisite to enrolling them in public schools. ${ }^{98}$ However, many states do allow for religious, and in some cases, philosophical exemptions to mandatory vaccines to accommodate the small percentage of the population who oppose vaccines due to personal beliefs. ${ }^{99}$ Mandatory vaccinations and the use of exemptions have gained considerable attention in the political sphere, and as a result mandating vaccines has become a dividing issue between those with different political ideologies. ${ }^{100-102}$ Some believe, as with many things in life, that it should ultimately be the right of an individual to determine what they put into their body and that the government should not be able to dictate their medical decisions. Other groups, however, would argue that government-mandated vaccination efforts are the only way to ensure herd immunity. The enactment of mandatory vaccination must be done with empathy and careful consideration, as numerous studies have shown that vaccination programs are much more successful when people willingly participate rather than being forced to do so by government mandates. After recent measles outbreaks in the US, some states responded by removing the personal belief exemption from school immunization requirements. ${ }^{103,104}$ It is worth noting that after California made this change, the number of medical exemptions in the state more than doubled. Additionally, children who are home schooled are not subjected to government-mandated vaccination, and thus it is unclear how many more children were vaccinated after the removal of personal belief exemptions. With these ideas in mind, public health officials may need to consider alternative methods to mandatory vaccination to increase the number of COVID-19 vaccine recipients. ${ }^{105}$

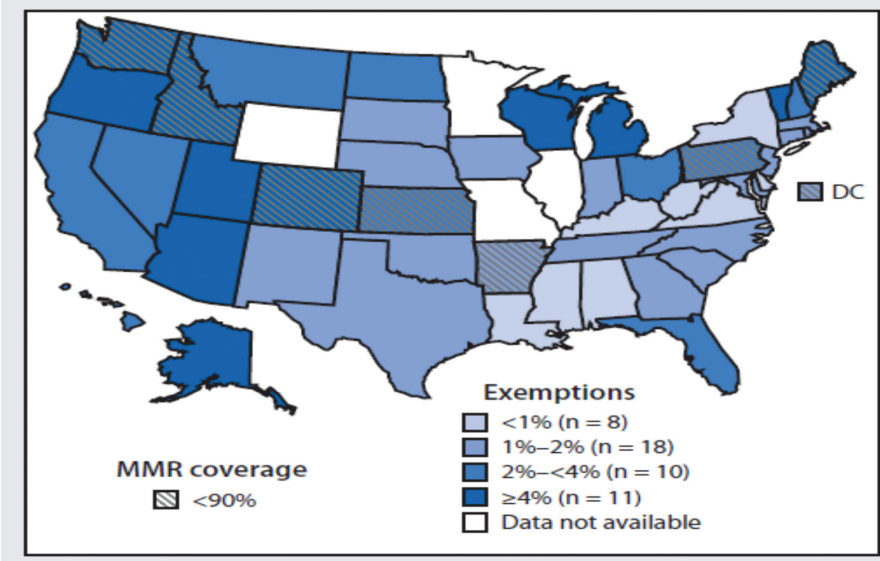

Figure 3. Estimated Percentage of Kindergarteners Who Were Exempt from One or More Vaccines 2013-2014 School Year. ${ }^{106}$

Figure 3 is a map of the United States showing the estimated percentage of children enrolled in kindergarten who have been exempted from receiving one or more vaccines and with $<90 \%$ coverage with 2 doses of measles, mumps, and rubella (MMR) vaccine in the United States during the 2013-14 school year. Among the 49 states and DC that reported 2013-14 school vaccination coverage, the median 2-dose MMR vaccination coverage was $94.7 \%$ (range $=81.7 \%$ in Colorado to $\geq 99.7 \%$ in Mississippi); 23 reported coverage $\geq 95 \%$, and eight reported coverage $<90 \%$. The percentage of kindergartners with an exemption was $<1 \%$ for eight states and $\geq 4 \%$ for 11 states (range $=<0.1 \%$ in Mississippi to $7.1 \%$ in Oregon), with a median of $1.8 \%$. https://www.cdc.gov/ $\mathrm{mmwr} / \mathrm{preview} / \mathrm{mmwrhtml} / \mathrm{mm} 6341 \mathrm{a} 1 . \mathrm{htm}$.

For a society to function smoothly, citizens must exhibit cooperation and a certain degree of respect for one another. There are many occasions during which individuals will take actions that have a greater benefit for the community than for themselves. These altruistic actions often lead to the success of communities. In times of danger those who are able are called upon to defend those who cannot protect themselves. There are people who, through no fault of their own, are unable to receive vaccinations due to biological factors or health conditions. When people choose 
to get vaccinated, they are not only protecting themselves but also indirectly protecting those who cannot be vaccinated through herd immunity. The recent measles outbreaks are a reminder that if an increasing proportion of the population does not get vaccinated, then the disease can make a comeback. ${ }^{51}$ It may encourage some people to know that by getting vaccinated, they are not only protecting themselves, but they are also protecting other vulnerable populations and contributing to eradicating certain preventable diseases. ${ }^{107}$

\section{LIMITATIONS}

The limitations of this review arose from the nature of the virus described, which is unique. Vaccine development for the novel coronavirus is an ongoing process involving a large network of government, charitable, and biopharmaceutical organizations. This results in a constant stream of new information regarding the COVID-19 vaccine being published and updated. Therefore, as it currently stands, the exact type, mechanisms of action, and projected availability of the vaccine is yet to be determined. In addition, since vaccine hesitancy lies along a spectrum and misinformation can be promoted through a variety of sources, it is difficult to determine what the specific nature of the concerns and oppositions to the vaccine will be.

\section{Conclusions}

Since their development, vaccines have proven to be effective tools for the prevention of numerous deadly diseases. ${ }^{1}$ Currently, there is no readily available vaccine for the novel coronavirus SARS-CoV-2, and the disease it causes, COVID-19. To prevent the continued spread of COVID-19, the ACTIV publicprivate partnership is working to safely develop and eventually administer the COVID-19 vaccine. ${ }^{21} \mathrm{~A}$ major obstacle to vaccine administration is vaccine hesitancy, which is often due to concerns with the necessity or safety of vaccines, although many other concerns also exist. ${ }^{22}$ Based upon the principles of direct, indirect, overall effect and health economics there is a definitive need for the use of vaccines. ${ }^{45}$ When the need for a particular vaccine has been established, a rigorous series of trials and regulations have been created to ensure safety and efficacy. Even after licensure, the monitoring of approved vaccines continues, and all adverse events can be reported and tracked. ${ }^{77,78}$ Vaccine hesitancy lies along a spectrum and can vary greatly between individuals and communities. Therefore, public health officials and healthcare providers should work closely with community leaders to correct misinformation and promote the benefits of life-saving vaccines. If healthcare workers can collaborate effectively with members of their respective communities to create population-specific strategies to overcome vaccine hesitancy, many lives may be saved, and future shutdowns avoided. . $^{96,101}$

Article citation: Perry C, Mizer A, Wynn A, Kruczek C. Countering COVID-19 vaccine hesitancy. The Southwest Respiratory and Critical Care Chronicles 2020;8(36):32-46 From: School of Medicine (CP, AM, AW, CK) and Department of Medical Education (CK), School of Medicine, Texas Tech University Health Sciences Center, Lubbock, Texas

Submitted: $7 / 30 / 2020$

Accepted: 9/15/2020

Reviewer: Kenneth Nugent MD

Conflicts of interest: none

This work is licensed under a Creative Commons Attribution-ShareAlike 4.0 International License.

\section{REFERENCES}

1. Stern AM, Markel H. The history of vaccines and immunization: familiar patterns, new challenges. Health Affairs 2005;24(3):611-21.

2. Ahn D-G, Shin H-J, Kim M-H, et al. Current status of epidemiology, diagnosis, therapeutics, and vaccines for novel coronavirus disease 2019 (COVID-19). J Microbiology Biotechnology 2020;30(3):313-24.

3. Gorbalenya, AE, Baker, SC, Baric, RS, et al. The species severe acute respiratory syndrome-related coronavirus: classifying 2019-nCoV and naming it SARS-CoV-2. Nat Microbiol 2020;5:536-544. https://doi.org/10.1038/ s41564-020-0695-z.

4. Sola I, Almazán F, Zúñiga S, et al. Continuous and discontinuous RNA synthesis in coronaviruses. Annual Rev Virology 2015;2(1):265-88. 
5. Fung TS, Liu DX. Human coronavirus: host-pathogen interaction. Annual Review of Microbiology 2019;73(1): $529-57$.

6. Li W, Shi Z, Yu M, et al. Bats are natural reservoirs of SARS-like coronaviruses. Science 2005;310(5748):676679. doi:10.1126/science.1118391.

7. Corman VM, Ithete NL, Richards LR, et al. Rooting the phylogenetic tree of Middle East Respiratory Syndrome coronavirus by characterization of a conspecific virus from an African bat. J Virology 2014;88(19):11297-303.

8. Zhou P, Yang X, Wang X, et al. A pneumonia outbreak associated with a new coronavirus of probable bat origin. Nature 2020;579:270-273. https://doi.org/10.1038/s41586020-2012-7.

9. Ji W, Wang W, Zhao X, et al. Cross species transmission of the newly identified coronavirus $2019 \mathrm{nCoV}$. J Medical Virology 2020;92(4):433-40.

10. Liu P, Chen W, Chen J-P. Viral metagenomics revealed Sendai virus and coronavirus infection of Malayan pangolins (Manis javanica). Viruses 2019;11(11):979.

11. Graham R, Donaldson E, Baric R. A decade after SARS: strategies for controlling emerging coronaviruses. Nat Rev Microbiol 2013;11:836-848. https://doi.org/10.1038/ nrmicro3143.

12. Peiris J, Lai S, Poon L, et al. Coronavirus as a possible cause of severe acute respiratory syndrome. The Lancet. 2003;361(9366):1319-25.

13. Yang $\mathrm{X}, \mathrm{Yu} \mathrm{Y}, \mathrm{Xu} \mathrm{J}$, et al. Clinical course and outcomes of critically ill patients with SARS-CoV-2 pneumonia in Wuhan, China: a single-centered, retrospective, observational study. The Lancet Respiratory Medicine 2020;8(5):47.

14. Zhang J-J, Dong X, Cao Y-Y, et al. Clinical characteristics of 140 patients infected with SARS CoV 2 in Wuhan, China. Allergy 2020.

15. About cloth face coverings [Internet]. Centers for Disease Control and Prevention. Centers for Disease Control and Prevention; 2020 [cited 2020Jun8]. Available from: https://www.cdc.gov/coronavirus/2019-ncov/preventgetting-sick/about-face-coverings.html.

16. California sof. stay home Q\&A [Internet]. ca.gov. [cited 2020Jun8]. Available from: https://covid19.ca.gov/stayhome-except-for-essential-needs/.

17. How to protect yourself \& others [Internet]. Centers for Disease Control and Prevention. Centers for Disease Control and Prevention; 2020 [cited 2020Jun8]. Available from: https://www.cdc.gov/coronavirus/2019-ncov/preventgetting-sick/prevention.html.

18. COVID-19 Map [Internet]. Johns Hopkins Coronavirus Resource Center. [cited 2020July14]. Available from: https://coronavirus.jhu.edu/map.html.
19. Kissler SM, Tedijanto C, Goldstein E, et al. Projecting the transmission dynamics of SARS-CoV-2 through the postpandemic period. Science 2020;368(6493):860-8.

20. Shaman J, Pitzer VE, Viboud C, et al. Absolute humidity and the seasonal onset of influenza in the continental United States. PLOS Biol 2010;8, e1000316. doi: 10.1371/ journal.pbio.1000316pmid:20186267.

21. Corey L, Mascola JR, Fauci AS, et al. A strategic approach to COVID-19 vaccine R\&D. Science 2020;368(6494): 948-50.

22. Deroo SS, Pudalov NJ, Fu LY. Planning for a COVID-19 vaccination program. JAMA 2020.

23. Expectations for a COVID-19 Vaccine [Internet]. APNORC.org | APNORC.org. [cited 2020Jun8]. Available from: http://www.apnorc.org/projects/Pages/Expectationsfor-a-COVID-19-Vaccine.aspx.

24. Edwards KM, Hackell JM. Countering vaccine hesitancy. Pediatrics 2016;138(3).

25. Thunstrom L, Ashworth M, Finnoff D, et al. Hesitancy towards a COVID-19 vaccine and prospects for herd immunity 2020. Available at http://dx.doi.org/10.2139/ ssrn.3593098.

26. Understanding how vaccines work [Internet]. Centers for Disease Control and Prevention. Centers for Disease Control and Prevention; 2018 [cited 2020Jun8]. Available from: https://www.cdc.gov/vaccines/hcp/conversations/ downloads/vacsafe-understand-color-office.pdf.

27. Vetter V, Denizer G, Friedland LR, et al. Understanding modern-day vaccines: what you need to know. Annals Medicine 2017;50(2):110-20.

28. Succi RCM. Vaccine refusal-what we need to know. J Pediatr (Rio J) 2018;94(6):574-581. doi: 10.1016/j.jped. 2018.01.008.

29. Lurie N, Saville M, Hatchett R, et al. Developing Covid-19 vaccines at pandemic speed. New England J Med 2020; 382(21):1969-73.

30. Huang J, Cao Y, Du J, et al. Priming with SARS CoV S DNA and boosting with SARS CoV S epitopes specific for CD4+ and $\mathrm{CD} 8+\mathrm{T}$ cells promote cellular immune responses. Vaccine 2007;25(39-40):6981-6991. doi: 10.1016/j.vaccine. 2007.06.047.

31. Lan J, Ge J, Yu J, et al. Structure of the SARS-CoV-2 spike receptor-binding domain bound to the ACE2 receptor. Nature 2020;581:215-220. https://doi.org/10.1038/ s41586-020-2180-5.

32. Zhu F-C, Li Y-H, Guan X-H, et al. Safety, tolerability, and immunogenicity of a recombinant adenovirus type- 5 vectored COVID-19 vaccine: a dose-escalation, openlabel, non-randomised, first-in-human trial. The Lancet 2020 . 
33. Khan KH. DNA vaccines: roles against diseases. Germs 2013;3(1):26-35. doi:10.11599/germs.2013.1034.

34. Deering RP, Kommareddy S, Ulmer JB, et al. Nucleic acid vaccines: prospects for non-viral delivery of mRNA vaccines. Expert opinion on drug delivery 2014;11(6):885-99.

35. Hobernik D, Bros M. DNA vaccines - how far from clinical use? International J Molecular Sciences 2018;19(11):3605.

36. Jones KL, Drane D, Gowans EJ. Long-term storage of DNA-free RNA for use in vaccine studies. BioTechniques 2007;43(5):675-81.

37. Stenler S, Blomberg P, Smith CE. Safety and efficacy of DNA vaccines. Human Vaccines \& Immunotherapeutics 2014;10(5):1306-8.

38. Liu. A comparison of plasmid DNA and mRNA as vaccine technologies. Vaccines 2019;7(2):37.

39. Routy JP, Boulassel MR, Yassine-Diab B, et al. Immunologic activity and safety of autologous HIV RNA-electroporated dendritic cells in HIV-1 infected patients receiving antiretroviral therapy. Clin Immunol 2010; 134:140-7. doi: 10.1016/j.clim.2009.09.009.

40. Allard SD, De Keersmaecker B, de Goede AL, et al. A phase I/IIa immunotherapy trial of HIV-1-infected patients with Tat, Rev and Nef expressing dendritic cells followed by treatment interruption. Clin Immunol 2012;142:25268. doi: 10.1016/j.clim.2011.10.010.

41. Gandhi RT, Kwon DS, Macklin EA, et al. Immunization of HIV-1-infected persons with autologous dendritic cells transfected with mRNA encoding HIV-1 Gag and Nef: results of a randomized, placebo-controlled clinical trial. J Acquir Immune Defic Syndr 2016;71:246-53. doi: 10.1097/QAI.0000000000000852.

42. Van Craenenbroeck AH, Smits EL, Anguille S, et al. Induction of cytomegalovirus-specific $\mathrm{T}$ cell responses in healthy volunteers and allogeneic stem cell recipients using vaccination with messenger RNA-transfected dendritic cells. Transplantation 2015;99:120-7. doi: 10.1097/ TP.0000000000000272.

43. Bahl K, Senn JJ, Yuzhakov O, et al. Preclinical and clinical demonstration of immunogenicity by mRNA vaccines against H10N8 and H7N9 influenza viruses. Mol Ther 2017;25:1316-27. doi: 10.1016/j.ymthe.2017.03.035.

44. Zhang C, Maruggi G, Shan H, et al. Advances in mRNA vaccines for infectious diseases. Frontiers Immunology 2019;10

45. Doherty M, Buchy P, Standaert B, et al. Vaccine impact: benefits for human health. Vaccine 2016;34(52):6707-14.

46. Beasely RP, Trepo C, Stevens CE, et al. The e antigen and vertical transmission of hepatitis B surface antigen, Am J Epidemiology 1977;105(Issue 2):94-98, https://doi.org/ 10.1093/oxfordjournals.aje.a112370j.
47. Edmunds WJ, Medley GF, Nokes DJ, et al. Hall epidemiological patterns of hepatitis B virus (HBV) in highly endemic areas. Epidemiol Infect 1996;117:313-325.

48. Shapiro CN. Epidemiology of hepatitis B. The Pediatric Infectious Disease Journal. 1993;12(5):433-7.

49. Poovorawan Y, Chongsrisawat V, Theamboonlers A, et al. Evidence of protection against clinical and chronic hepatitis $\mathrm{B}$ infection 20 years after infant vaccination in a high endemicity region. Journal of Viral Hepatitis 2011; 18(5):369-75.

50. Vaccination greatly reduces disease, disability, death and inequity worldwide [Internet]. World Health Organization. World Health Organization; 2011. Available from: https:// www.who.int/bulletin/volumes/86/2/07-040089/en/.

51. Fine $P$, Eames K, Heymann DL. Herd immunity: a rough guide. Clin Infect Dis 2011;52(7):911-916. doi:10.1093/ cid/cir007.

52. Kretzschmar M, Teunis PFM, Pebody RG. Incidence and reproduction numbers of pertussis: estimates from serological and social contact data in five European countries. PLoS Medicine 2010;7(6).

53. Plans-Rubió P. Evaluation of the establishment of herd immunity in the population by means of serological surveys and vaccination coverage. Hum Vaccin Immunother 2012;8:184-188, 10.4161/hv.18444.

54. Paulke-Korinek M, Kundi M, Rendi-Wagner P, et al. Herd immunity after two years of the universal mass vaccination program against rotavirus gastroenteritis in Austria. Vaccine 2011;29(15):2791-6.

55. Hanquet $G$, Valenciano $M$, Simondon $F$, et al. Vaccine effects and impact of vaccination programmes in postlicensure studies Vaccine 2013;31:5634-5642, 10.1016/j. vaccine.2013.07.006.

56. Impact of vaccines in the 20 th $\& 21$ st centuries [Internet]. Center for Disease Control and Prevention. 2019 [cited 2020Jun8]. Available from: https:/www.cdc.gov/vaccines/ pubs/pinkbook/downloads/appendices/e/impact.pdf.

57. Roush SW. Historical comparisons of morbidity and mortality for vaccine-preventable diseases in the United States. JAMA 2007;298(18):2155.

58. Notifiable infectious diseases and conditions data tables [Internet]. Centers for Disease Control and Prevention. Centers for Disease Control and Prevention; [cited 2020Jun8]. Available from: https://wwwn.cdc.gov/nndss/ infectious-tables.html.

59. Evidence supporting transmission of severe acute respiratory syndrome coronavirus 2 while presymptomatic or asymptomatic. Emerging Infectious Diseases J - Centers for Disease Control and Prevention 2020;26(7). Available from: https://wwwnc.cdc.gov/eid/article/26/7/20-1595_articlel 
60. Eastern Mediterranean region [Internet]. World Health Organization. World Health Organization; [cited 2020Jun23]. Available from: http://www.emro.who.int/ health-topics/corona-virus/transmission-of-covid-19-byasymptomatic-cases.html.

61. Gandhi M, Yokoe DS, Havlir DV. Asymptomatic transmission, the Achilles' heel of current strategies to control Covid-19. New England Journal of Medicine 2020; 382(22):2158-60.

62. Bai Y, Yao L, Wei T, et al. Presumed asymptomatic carrier transmission of COVID-19. JAMA 2020;323(14):1406.

63. News Release Bureau of Labor Statistics [Internet]. U.S. Department of Labor. 2020 [cited 2020Jun]. Available from: https://www.bls.gov/news.release/pdf/empsit.pdf.

64. Nicola M, Alsafi Z, Sohrabi C, et al. The socio-economic implications of the coronavirus pandemic (COVID-19): a review. International J Surgery 2020;78:185-93.

65. Vaccines: Vac-Gen/what would happen if we stopped vaccinations [Internet]. Centers for Disease Control and Prevention. Centers for Disease Control and Prevention; 2018 [cited 2020Jun8]. Available from: https://www.cdc.gov/ vaccines/vac-gen/whatifstop.html.

66. Mariani L, Venuti A. HPV vaccine: an overview of immune response, clinical protection, and new approaches for the future. J Translational Med 2010;8(1).

67. Wendelboe AM, Rie AV, Salmaso S, et al. Duration of immunity against pertussis after natural infection or vaccination. The Pediatric Infectious Disease J 2005;24(Supplement).

68. Christenson B, Böttiger M. Measles antibody: comparison of long-term vaccination titres, early vaccination titres and naturally acquired immunity to and booster effects on the measles virus. Vaccine 1994;12(2):129-33.

69. Plotkin SA, Orenstein WA, Offit PA. Plotkin's vaccines. Philadelphia, PA: Elsevier; 2018.

70. Sherrid, A, Ruck, C, Sutherland, D. Lack of broad functional differences in immunity in fully vaccinated vs. unvaccinated children. Pediatr Res 2017;81:601-608.

71. Robbiani DF, Gaebler C, Muecksch F, et al. Convergent antibody responses to SARS-CoV-2 in convalescent individuals. Nature 2020. https://doi.org/10.1038/s41586-0202456-9.

72. Nabel, Gary J. Designing tomorrow's vaccines. The New England Journal of Medicine, U.S. National Library of Medicine 2013. www.ncbi.nlm.nih.gov/pmc/articles/PMC3612922/.

73. Tanne, JH. MMR vaccine is not linked with autism, says Danish study 2002. www.ncbi.nlm.nih.gov/pmc/articles/ PMC1124634/.

74. Taylor LE, et al. Vaccines are not associated with autism: an evidence-based meta-analysis of case-control and cohort studies. Vaccine U.S. National Library of Medicine. pubmed.ncbi.nlm.nih.gov/24814559/.

75. Vaccines do not cause autism [Internet]. Centers for Disease Control and Prevention. Centers for Disease Control and Prevention; 2020 [cited 2020Jun9]. Available from: https://www.cdc.gov/vaccinesafety/concerns/autism.html.

76. Cunningham A, Garçon N, Leo O, et al. Vaccine development: from concept to early clinical testing. 2016. Retrieved July 13, 2020, from https://www.sciencedirect. com/science/article/pii/S0264410X16309173.

77. Mitchell VS, Philipose NM, Sanford JP. The children's vaccine initiative: achieving the vision. Institute of Medicine (US) Committee on the Children's Vaccine Initiative: Planning Alternative Strategies; Washington (DC): National Academies Press (US). 1993. Available from: https://www. ncbi.nlm.nih.gov/books/NBK236428/.

78. Vaccine testing and approval process [Internet]. Centers for Disease Control and Prevention. Centers for Disease Control and Prevention. 2014 [cited 2020Jun9]. Available from: https://www.cdc.gov/vaccines/basics/test-approve.html.

79. Vaccine safety datalink (VSD) [Internet]. Centers for Disease Control and Prevention. Centers for Disease Control and Prevention; 2019 [cited 2020Jun9]. Available from: https://www.cdc.gov/vaccinesafety/ensuringsafety/monitoring/vsd/index.html.

80. Chen RT, Davis RL, Rhodes PH. Special methodological issues in pharmacoepidemiology studies of vaccine safety. Pharmacoepidemiology 4th ed. 2006. doi: 1002/ 9780470059876.

81. Chen RT, Rastogi SC, Mullen JR, et al. The vaccine adverse event reporting system (VAERS). Vaccine 1994;12(6): 542-50. doi: 10.1016/0264-410X(94)90315-8.

82. Miller ER, Suragh T, Hibbs B, et al. Surveillance manual [Internet]. Centers for Disease Control and Prevention. Centers for Disease Control and Prevention; 2017 [cited 2020Jul3]. Available from: https://www.cdc.gov/vaccines/ pubs/surv-manual/chpt21-surv-adverse-events.html.

83. Mullard A. COVID-19 vaccine development pipeline gears up 2020. www.ncbi.nlm.nih.gov/pmc/articles/PMC7272155/.

84. Plotkin S, Robinson JM, Cunningham G, et al. The complexity and cost of vaccine manufacturing - an overview. 2017. www.ncbi.nlm.nih.gov/pmc/articles/PMC5518734/.

85. Smith J, Lipsitch M, Almond JW, et al. Vaccine production, distribution, access, and uptake. Lancet (London, England), U.S. National Library of Medicine 2011. www.ncbi. nlm.nih.gov/pmc/articles/PMC3164579/.

86. Abbasi J, Fauci A. on COVID-19 vaccines, schools. In: JAMA 2020. https://jamanetwork.com/journals/jama/ fullarticle/2767208. Accessed 10 Jun 2020. 
87. DeStefano F, Shimabukuro TT. The MMR vaccine and autism. Annu Rev Virol 2019;6(1):585-600. doi:10.1146/ annurev-virology-092818-015515.

88. Farrington CP, Miller E, Taylor B. MMR and autism: further evidence against a causal association. Vaccine 2001;19(27): 3632-3635. doi:10.1016/s0264-410x(01)00097-4.

89. Dubé E, Vivion M, Macdonald NE. Vaccine hesitancy, vaccine refusal and the anti-vaccine movement: influence, impact and implications. Expert Review Vaccines 2014; 14(1):99-117.

90. Trogen B, Oshinsky D, Caplan A. Adverse consequences of rushing a SARS-CoV-2 vaccine. JAMA 2020;323(24):2460.

91. Keelan J, Pavri-Garcia V, Tomlinson G, et al. YouTube as a source of information on immunization: a content analysis. JAMA 2007;298(21):2482-2484. doi:10.1001/jama. 298.21.2482.

92. Leask J, Should we do battle with antivaccination activists? Public Health Res Pract 2015;25(2):e2521515. doi:10.17061/phrp2521515.

93. Horne Z, Powell D, Hummel JE, et al. Countering antivaccination attitudes. Proc Natl Acad Sci USA 2015;112(33): 10321-10324. doi:10.1073/pnas.1504019112.

94. Yancy CW. COVID-19 and African Americans. JAMA 2020;323(19):1891-1892. doi:10.1001/jama.2020.6548.

95. COVID-19 in racial and ethnic minority groups [Internet]. Centers for Disease Control and Prevention. Centers for Disease Control and Prevention; 2020 [cited 2020Jul20]. Available from: https://www.cdc.gov/coronavirus/2019ncov/need-extra-precautions/racial-ethnic-minorities.html.

96. Ward PR. Improving access to, use of, and outcomes from public health programs: the importance of building and maintaining trust with patients/clients. Front Public Health 2017;5:22. doi:10.3389/fpubh.2017.00022.

97. Wooten KG, Luman ET, Barker LE. Socioeconomic factors and persistent racial disparities in childhood vaccination. Am J Health Behav 2007;31(4):434-445. doi:10.5555/ ajhb.2007.31.4.434.
98. MacDonald NE, Harmon S, Dube E, et al. Mandatory infant \& childhood immunization: rationales, issues and knowledge gaps. Vaccine 2018;36(39):5811 5818. doi: 10.1016/j.vaccine. 2018.08 .042 .

99. Bazin $H$. The ethics of vaccine usage in society: lessons from the past. Endeavour 2001;25(3):104-108. doi:10.1016/s0160-9327(00)01376-4.

100. Ciolli A. Mandatory school vaccinations: the role of tort law. Yale J Biol Med 2008;81(3):129-137.

101. Baumgaertner B, Carlisle JE, Justwan F. The influence of political ideology and trust on willingness to vaccinate. Plos One 2018;13(1).

102. McCoy, Charles. Anti-vaccination beliefs do not follow the usual political polarization. The Conversation [Internet]. 2017. Available from: https://theconversation.com/anti-vaccinationbeliefs-dont-follow-the-usual-political-polarization-81001.

103. Rabinowitz M, Latella L, Stern $C$, et al. Beliefs about childhood vaccination in the United States: political ideology, false consensus, and the illusion of uniqueness. PLoS One 2016;11(7):e0158382. doi: 10.1371/journal.pone.0158382.

104. Majumder MS, Cohn EL, Mekaru SR, et al. Substandard vaccination compliance and the 2015 Measles outbreak. JAMA Pediatr 2015;169(5):494-495. doi:10.1001/ jamapediatrics.2015.0384.

105. McDonald P, Limaye RJ, Omer SB, et al. Exploring California's new law eliminating personal belief exemptions to childhood vaccines and vaccine decision-making among homeschooling mothers in California. Vaccine 2019;37(5):742 750. doi: 10.1016/j.vaccine.2018.12.018.

106. Vaccination coverage among children in kindergartenUnited States, 2013-14 School Year [Internet]. Centers for Disease Control and Prevention. Centers for Disease Control and Prevention; [cited 2020Jun23]. Available from: https:// www.cdc.gov/mmwr/preview/mmwrhtml/mm6341a1.htm.

107. Hussain A, Ali S, Ahmed M, et al. The Anti-vaccination movement: a regression in modern medicine. Cureus 2018; 10(7):e2919. doi:10.7759/cureus.2919. 